\title{
Specimen records for North American Lepidoptera (Insecta) in the Oregon State Arthropod Collection. Zygaenoidea: Zygaenidae, Latreille 1809, Limacodidae, Moore 1879, Dalceridae Dyar, 1898 and Megalopygidae Herrich-Schäffer, 1855
}

Jon H. Shepard

Paul C. Hammond

Christopher J. Marshall

Oregon State Arthropod Collection, Department of Integrative Biology, Oregon State University, Corvallis OR 97331

Cite this work, including the attached dataset, as:

Shepard, J. H., P. C. Hammond, C. J. Marshall. 2019. Specimen records for North American Lepidoptera (Insecta) in the Oregon State Arthropod Collection. Zygaenoidea: Zygaenidae, Latreille 1809, Limacodidae, Moore 1879, Dalceridae Dyar, 1898 and Megalopygidae Herrich-Schäffer, 1855. Catalog: Oregon State Arthropod Collection 3(2) (beta version). http://dx.doi.org/10.5399/osu/cat osac.3.2.4593

\section{Introduction}

These records were generated using funds from the LepNet project (Seltmann et. al., 2017) - a national effort to create digital records for North American Lepidoptera. The dataset published herein contains the label data for all North American specimens of Zygaenidae, Limacodidae, Dalceridae and Megalopygidae residing at the Oregon State Arthropod Collection as of March 2019. A beta version of these data records will be made available on the OSAC server (http: / / osac.oregonstate.edu/IPT) at the time of this publication. The beta version will be replaced in the near future with an official release (version 1.0), which will be archived as a supplemental file to this paper.

\section{Methods}

Basic digitization protocols and metadata standards can be found in (Shepard et al. 2018). Identifications were confirmed prior to determination by Jon Shepard and Paul Hammond using the online Digital Guide to Moth Identification website (Moth Photographers Group, 2019). Nomenclature was confirmed using the species matching tool provided by GBIF (https:// www.gbif.org/tools/species-lookup).

\section{Results}

The collection has only 232 specimens of North American Zygaenoidea, the vast majority of which (204) belong to the family Limacodidae. The genera present are: Dalceridae: Dalcerides Neumoegen and Dyar, 1893; Limacodidae: Euclea Hübner, 1819, Isa Packard, 1864, Lithacodes Packard, 1864, Monoleuca Grote \& Robinson, 1868, Parasa Moore, 1859, Prolimacodes Schaus, 1896, Tortricidia Packard, 1864; Megalopygidae: Megalopyge Hübner, 1820; and Zygaenidae: Harrisina Packard, 1864 (Table 1). 
Table 12. Species inventory of OSAC North American Zygaenoidea

Family

Taxon

\# specimens

\begin{tabular}{|l|l|c|}
\hline Dalceridae & Dalcerides ingenita Edwards, 1882 & 1 \\
\hline Limacodidae & Euclea delphinii Boisduval, 1832 & 19 \\
\hline & Euclea obliqua Edwards, 1886 & 3 \\
\hline & Isa schaefferana Dyar, 1905 & 2 \\
\hline & Lithacodes fasciola Herrich-Schäffer, 1854 & 2 \\
\hline & Monoleuca occidentalis Barnes \& McDunnough, 1912 & 6 \\
\hline & Parasa chloris Herrich-Schäffer, 1854 & 3 \\
\hline & Prolimacodes badia Hübner, 1835 & 3 \\
\hline & Prolimacodes trigona Edwards, 1882 & 9 \\
\hline & Tortricidia flexuosa Grote, 1880 & 2 \\
\hline & Tortricidia testacea Packard, 1864 & 155 \\
\hline Megalopygidae & Megalopyge crispata Packard, 1864 & 2 \\
\hline & Megalopyge opercularis Smith \& Abbot, 1797 & 5 \\
\hline Zygaenidae & Harrisina metallica Stretch, 1885 & 20 \\
\hline & & total \\
\hline
\end{tabular}

Additional material from outside of North America is present, but was not digitized and catalogued at this time.

Label data for these specimens can be accessed via online data portals that serve osac IPT data (e.g., www.gbif.org) or by direct download via the archived datasets stored in the supplemental files of this paper.

\section{Discussion}

Owing to their relative scarcity in North America and the Pacific Northwest it is not surprising that the OSAC possesses few examples of the Zygaenoidea. The vast majority of the holdings belong to the family Limacodidae, and a single species in particular: Tortricidia testacea Packard, 1864 which is common in the Pacific Northwest. Only a single example of the family Dalceridae is present, and only 7 examples of the Megalopygidae which are from the American southwest. Although Oregon has a native zygaenid: Harrisina metallica Stretch, 1885, the 20 specimens in the collection were obtained in California or elsewhere outside of the Pacific Northwest. The species is reported in southwestern Oregon where it can be a pest on grapes and vineyards. Voucher material from Oregon should be targeted in the future to ensure the local populations are represented.

Minor corrections and other small modifications made in the future to this dataset will be accomplished by issuing a new version and providing a file of modified records to the supplemental files. Major additions, such as new records, new data fields, new contributors or other significant modifications will be accomplished through the generation and release of new datasets. 


\section{Acknowledgments}

The records published herein were generated with funds from NSF: DBI\# 1601888: Digitization TCN: Collaborative Research: Lepidoptera of North America Network: Documenting Diversity in the Largest Clade of Herbivores.

\section{References}

Moth Photographers Group (2019) Digital Guide to Moth Identification. https:/ / mothphotographersgroup.msstate.edu [accessed April 1, 2019].

Seltmann, K. C. et al. 2017. LepNet: The Lepidoptera of North America Network. Zootaxa 4247 (1): 073-077. https:/ / doi.org/10.11646/ zootaxa.4247.1.10.

Shepard J.H., C.J. Marshall, P.C. Hammond. 2018. North American Lepidoptera in the Oregon State Arthropod Collection: Hesperiidae, Pieridae, Papilionidae. Catalog: Oregon State Arthropod Collection 2(2): 1-2 and supplement file. doi: $\underline{\text { http: / / dx.doi.org/10.5399/osu/cat osac.2.2.4480 }}$ 